\title{
Comparison of the roll-plate and sonication techniques in the diagnosis of microbial ureteral stent colonisation: results of the first prospective randomised study
}

\author{
G. Bonkat • O. Braissant $\cdot$ M. Rieken • G. Müller $\cdot$ R. Frei • \\ Andre van der Merwe · F. P. Siegel • T. C. Gasser • \\ S. Wyler $\cdot$ A. Bachmann $\cdot$ A. F. Widmer
}

Received: 22 August 2012/ Accepted: 22 September 2012/Published online: 5 October 2012

(c) Springer-Verlag Berlin Heidelberg 2012

\begin{abstract}
Background Microbial ureteral stent colonisation (MUSC) is one leading risk factor for complications associated with ureteral stent placement. As MUSC remains frequently undetected by standard urine cultures, its definitive diagnosis depends on microbiological investigation of the stent. However, a standard reference laboratory technique for studying MUSC is still lacking.

Materials and methods A total of 271 ureteral stents removed from 199 consecutive patients were investigated. Urine samples were obtained prior to device removal. Stents were divided into four parts. Each part was separately processed by the microbiology laboratory within $6 \mathrm{~h}$. Ureteral stents were randomly allocated to roll-plate or sonication, respectively, and analysed using standard
\end{abstract}

G. Bonkat $(\bowtie) \cdot$ O. Braissant $\cdot$ M. Rieken · G. Müller ·

T. C. Gasser · S. Wyler · A. Bachmann

Department of Urology, University Hospital Basel,

Spitalstrasse 21, 4031 Basel, Switzerland

e-mail: bonkatg@uhbs.ch

R. Frei

Clinical Microbiology Laboratory, University Hospital Basel,

Spitalstrasse 21, 4031 Basel, Switzerland

A. van der Merwe

Department of Urology, Faculty of Health Sciences, University of Stellenbosch, PO Box 19063, Tygerberg 7505, South Africa

F. P. Siegel

Department of Urology, University Hospital Mannheim, Ruprecht-Karls University of Heidelberg, Theodor-Kutzer-Ufer 1-3, 68167 Mannheim, Germany

\section{A. F. Widmer}

Division of Infectious Diseases and Hospital Epidemiology, University Hospital Basel, Petersgraben 4, 4031 Basel, Switzerland microbiological techniques. Demographic and clinical data were prospectively collected using a standard case-report form.

Results Overall, roll-plate showed a higher detection rate of MUSC compared with sonication ( 35 vs. $28 \%, p<0.05$ ) and urine culture ( 35 vs. $8 \%, p<0.05$ ). No inferiority of Maki's technique was observed even when stents were stratified according to indwelling time below or above 30 days. Compared with roll-plate, sonication commonly failed to detect Enterococcus spp., coagulase-negative staphylococci (CoNS) and Enterobacteriaceae. In addition, sonication required more hands-on time, more equipment and higher training than roll-plate in the laboratory.

Conclusions This prospective randomised study demonstrates the superiority of Maki's roll-plate technique over sonication in the diagnosis of MUSC and that urine culture is less sensitive than both methods. The higher detection rate, simplicity and cost-effectiveness render roll-plate the methodology of choice for routine clinical investigation as well as basic laboratory research.

Keywords Biofilm - Maki's technique $\cdot$ Microbial ureteral stent colonisation $\cdot$ Roll-plate $\cdot$ Sonication

\section{Introduction}

Ureteral stent placement is a fundamental part of daily urological practice. The procedure is simple and well tolerated in most cases. However, associated complications such as urinary tract infection, stent blockage and ureteral stentrelated symptoms develop frequently and may be intense [1, 2]. Microbial ureteral stent colonisation (MUSC) is considered to be an important etiological factor in the pathogenesis of these complications [3-5]. In an attempt to prevent 
MUSC, extensive research has been directed towards ureteral stent modifications, including silver-, heparin- or diamond-coated surfaces, triclosan-eluting stents, controlled release of antibiotics and surface modifications to change hydrophobicity [6-9]. In this context, it is obvious that a solid understanding of the pathogens involved is the key for the development of successful MUSC-preventive innovations. However, currently no standard reference method for the detection of MUSC exists. Previous studies analysing MUSC used either sonication [10-14] or broth culture [15], while other studies did not provide a detailed description of the methodology used [5, 16-18]. Interestingly, Maki 's rollplate technique [19], the international reference and most widely used technique for the diagnosis of catheter-related blood stream infections (CRBSI), has not been commonly reported [20]. This semiquantitative technique, also called the roll-plate method, consists of rolling the catheter tip back and forth over the surface of an agar plate. Since roll-plate is not inferior to sonication in the detection of CRBSI [21, 22], its application for the diagnosis of MUSC may be favourable. The objective of this prospective randomised study was to compare sonication to roll-plate technique in the diagnosis of MUSC and to define the procedure of choice for both routine microbiological investigation and basic laboratory research.

\section{Materials and methods}

\section{Study population}

All consecutive patients who had a ureteral stent removed in our department during the period from 1 July 2009 to 23 July 2010 were eligible for study participation. The study was approved by the local human research ethics committee and complied with the provisions of the Declaration of Helsinki, Good Clinical Practice guidelines and local laws and regulations. All included patients gave written informed consent.

\section{Laboratory investigations}

Ureteral stents were removed under aseptic conditions by cystoscopy. To exclude an experimental bias due to exclusive proximal or distal colonisation, stents were divided into four parts. Each part was separately placed into a sterile tube and processed by the microbiology laboratory within $6 \mathrm{~h}$. The two proximal and distal specimens were randomly assigned for roll-plate or sonication (Fig. 1) by using computer-generated numbers. Urine specimens were obtained prior to stent removal via single catheterisation or midstream clean catch technique. Specimens were plated using calibrated sterile wire loops that deliver a known volume of urine $(1 \mu \mathrm{l})$ and analysed by conventional culture methods, outlined by the Manual of Clinical Microbiology, ASM, following guidelines issued by the Clinical and Laboratory Standards Institute (CLSI). The roll-plate method was performed by rolling the external surface of a catheter tip back and forth on the surface of a Columbia agar plate supplemented with $5 \%$ sheep blood (BD, Franklin Lakes, NJ) at least three times and then incubating the plate for $72 \mathrm{~h}$ at $5 \% \mathrm{CO}_{2}$ and $37{ }^{\circ} \mathrm{C}$, after which the number of colony-forming units (CFU) was quantitated. Stent colonisation was detected by sonication as described previously [3, 10, 11].

\section{Definitions}

\section{Sonication positive}

Microbial growth of $\geq 100 \mathrm{CFU} / \mathrm{ml}$ in sonicate-fluid culture [23].

\section{Roll-plate positive}

Positive semiquantitative tip culture of $\geq 15$ CFU [19].

\section{Positive urine culture}

Urine cultures were interpreted according to Wilson et al. [24] regarding collecting technique (i.e. single catheterisation, midstream clean catch technique), quantitation ( $\geq 100 \mathrm{CFU} / \mathrm{ml}$ ) and the number of microorganisms isolated.

\section{Statistical analysis}

The sample size and power calculation were based on published data on MUSC [10, 20]. With an adjusted, estimated rate of MUSC of $50 \%$ in the roll-plate group and

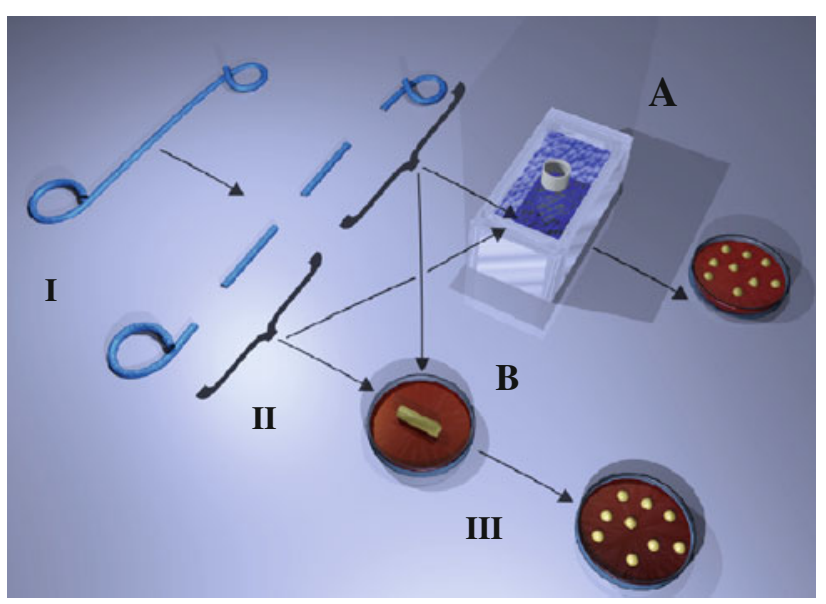

Fig. 1 Experimental set-up: I, stents were divided into four parts; II, the two proximal and distal specimens were randomly assigned for sonication $\mathbf{a}$ or roll-plate $\mathbf{b}$; III, roll-plate and sonicate-fluid culture 
with a power of $90 \%$ to show a difference of $12 \%$ to sonication, 212 ureteral stents were necessary $(\alpha=0.05$, error $\beta=0.10)$. McNemar's test was applied as appropriate. A two-tailed $p$ value of $<0.05$ was considered to indicate statistical significance. Statistical analysis was computed on Statistical Package for Social Sciences (SPSS, version 20.00; Chicago, IL, USA) for Windows ${ }^{\mathrm{TM}}$.

\section{Results}

General characteristics

During the study period, 271 ureteral stents were removed from 199 consecutive patients (62\% male, median age 56, range 24-74 years). The median ureteral stent indwelling time was 42 days (range 4-392 days). After initial laboratory preparation, 1,084 stent segments were investigated.

\section{Roll-plate versus sonication}

Overall, roll-plate showed a higher detection rate of MUSC compared with sonication (35 vs. $28 \%, p<0.05)$. Twenty-four discordant observational pairs were positive with roll-plate only, compared with five positives with sonication only (Table 1). Thirteen ureteral stents positive with roll-plate required subcultivation due to multiple microbial growths. After stratification according to the length of indwelling time, roll-plate proved to detect statistically significant more colonised stents compared with sonication in long-term ( $n=171,37$ vs. $29 \%, p<0.05)$ but not in short-term ( $n=100,33$ vs. $27 \%, p=0.14$ ) indwelling stents.

\section{Patients with bilateral stents}

Thirty stents were obtained from patients with bilateral placement. Of these 15 pairs, $13(87 \%)$ and $10(67 \%)$ showed identical results using sonication and roll-plate, respectively. All stents positive with sonication were positive with roll-plate and revealed identical microbial growth.

\section{Site of MUSC}

No significant differences regarding the site of MUSC using both techniques were observed. Of the 96 roll-platepositive ureteral stents, $70(73 \%)$ were proximal and distal colonised, while 26 stents showed only distal $(n=13$, $13.5 \%)$ or proximal $(n=13,13.5 \%)$ microbial growth. Similarly, $52(67 \%)$ of 77 sonication-positive ureteral stents were proximal and distal colonised, whereas only proximal colonisation was found in $12(16 \%)$ cases and only distal colonisation in $13(17 \%)$ cases, respectively.
Table 1 Diagnostic yield of roll-plate versus sonication

\begin{tabular}{|c|c|c|c|c|}
\hline \multirow[t]{3}{*}{ Sonication } & \multicolumn{4}{|l|}{ A) Overall } \\
\hline & \multicolumn{4}{|l|}{ Roll-plate } \\
\hline & Positive & Negative & & \\
\hline Positive & $72(26 \%)$ & $5(2 \%)$ & $77(28 \%)$ & $p<0.05$ \\
\hline \multirow[t]{2}{*}{ Negative } & $24(9 \%)$ & $170(63 \%)$ & $194(72 \%)$ & \\
\hline & $96(35 \%)$ & $175(65 \%)$ & $271(100 \%)$ & \\
\hline \multirow[t]{3}{*}{ Sonication } & \multicolumn{4}{|c|}{ B) Indwelling time $<30 \mathrm{~d}$} \\
\hline & \multicolumn{4}{|l|}{ Roll-plate } \\
\hline & Positive & Negative & & \\
\hline Positive & $24(24 \%)$ & $3(3 \%)$ & $27(27 \%)$ & $p<0.14$ \\
\hline \multirow[t]{2}{*}{ Negative } & $9(9 \%)$ & $64(64 \%)$ & $63(63 \%)$ & \\
\hline & $33(33 \%)$ & $67(67 \%)$ & $100(100 \%)$ & \\
\hline \multirow[t]{3}{*}{ Sonication } & \multicolumn{4}{|c|}{ c) Indwelling time $\geq 30 \mathrm{~d}$} \\
\hline & \multicolumn{4}{|l|}{ Roll-plate } \\
\hline & Positive & Negative & & \\
\hline Positive & $48(28 \%)$ & $2(1 \%)$ & $50(29 \%)$ & $p<0.05$ \\
\hline \multirow[t]{2}{*}{ Negative } & $15(9 \%)$ & $106(62 \%)$ & $121(71 \%)$ & \\
\hline & $63(37 \%)$ & $108(63 \%)$ & $171(100 \%)$ & \\
\hline
\end{tabular}

Cut-offs used for detection of microbial ureteral stent colonisation: roll-plate method ( $\geq 15 \mathrm{CFU})$, sonication $(\geq 100 \mathrm{CFU} / \mathrm{ml})$

Roll-plate and sonication versus urine culture

In comparison with urine culture, both roll-plate (35 vs. $8 \%, p<0.05)$ and sonication (28 vs. $8 \%, p<0.05)$ resulted in a significantly higher detection rate of MUSC.

Microorganisms

A total of 306 microorganisms were isolated (172 using roll-plate and 134 using sonication). Applying a cut-off $\geq 15 \mathrm{CFU}$, roll-plate detected 138 microorganisms compared with 109 detected by sonication (cut-off $\geq 100 \mathrm{CFU} / \mathrm{ml}$ ).

Roll-plate and sonication observed similar rates of single microbial growth (68 vs. $66 \%$ ) and multiple growths (32 vs. $34 \%$ ), respectively. Most commonly isolated by roll-plate were Enterococcus spp. (24\%), followed by coagulase-negative staphylococci (CoNS) (17\%) and Enterobacteriaceae (16\%), whereas sonication predominantly detected Enterococcus spp. (21\%), Candida spp. (17\%) and Enterobacteriaceae (15\%) (Fig. 2). Twentyseven organisms on 24 ureteral stents were identified by roll-plate only, compared with 5 organisms on 5 stents positive by sonication only $(p<0.05)$. Thirty-one organisms detected on 13 ureteral stents by roll-plate required 
sub cultivation of primary culture. The spectrum of microorganisms identified in cases with positive roll-plate and positive sonication was identical in $78 \%$ of the cases. In the remaining $22 \%$, similar microbial growth was detected with (1) additional organisms in roll-plate $(13 \%)$ or (2) sonication (6\%) and (3) different additional organisms $(3 \%)$, respectively.

\section{Discussion}

This prospective randomised study is, to our knowledge, the first of its kind and demonstrates the superiority of Maki's roll-plate technique over sonication in the investigation of MUSC. The following are the advantages of roll-plate: (1) a higher detection rate, (2) identification of a greater number of microorganisms, (3) no need for additional technical equipment (i.e. ultrasound bath, hydrophone), (4) time saving ( 2 vs. $10 \mathrm{~min}$ ), and (5) cost-efficiency. With respect to the results of this study, the frequent use of sonication in previous studies investigating MUSC might be regarded as too uncritical, as its presence may have been underestimated [3, 10-13, 25].

The disadvantage of roll-plate is that the detection of multiple microbial growths could be interpreted as contamination, and sub cultivation may be necessary. The higher sensitivity of sonication to detect inner and outer surface microorganisms could not be proved in our study. Despite some limitations of sonication, it detected at least $75 \%$ of roll-plate-positive cases in this study, and in five cases, only sonication was positive. In addition, sonication is more efficient than roll-plate in identifying mixed biofilms as subcultivation was not necessary in most cases. Therefore, sonication remains a valuable tool for bench research of mixed biofilms. In either case, sonication is more reliable than simple culture of ureteral stents in broth [15]. The disadvantages of using liquid media (broth) include (1) overgrowth of other species by fast-growing organisms and (2) the inability to quantify CFUs [26].

At this point, it should be mentioned that the cut-offs used for sonication and roll-plate in this study are the standard values for central line catheters to identify catheter-related blood stream infections [23]. Perhaps these values might not be the ideal cut-offs for diagnosing MUSC, but with increasing number of studies, such values will certainly be refined [3]. As the urinary tract is highly susceptible to infection once microorganisms gain access to it, only small numbers of pathogens might be clinically relevant. Therefore, higher cut-offs should be considered with caution.

Regarding the microorganisms identified in this study, it is interesting that especially Gram-positive organisms such as Enterococcus spp. and CoNS were missed by sonication. This indicates that sonication is not able to sufficiently liberate organisms which form thick, multilayered biofilms. The major drawbacks of both techniques are that ureteral stents have to be removed for diagnosis and that only mechanically detached organisms can be detected. Although there is growing evidence that MUSC may be the cause of ureteral stent-related symptoms, such as urgency and frequency $[3,9]$, the role of MUSC as origin of urinary tract infections (UTI) remains highly speculative. Theoretically, MUSC organisms could be shed into the urine during manipulation or instrumentation, but so far no study has proved this theory. Although many reports have cited risk factors for the development of UTI after manipulation of indwelling ureteral stents (e.g. positive preoperative urine culture, previous treatment with antibiotics, immunocompromised states, prolonged indwelling time), most of them have not investigated the stent and therefore questioned the role of MUSC. For example, Gautam et al. described fungal urosepsis after extended endoscopic treatment (i.e. ureteroscopy followed by percutaneous nephrolithotomy) of steinstrasse in a patient with a forgotten ureteral stent, but did not investigate the stent for the presence of the causative organism [4]. Similarly, Gross et al. did not study the stents in their report about two elderly patients who developed Candida albicans sepsis $12 \mathrm{~h}$ after ureteroscopy and ureteral stenting for obstructing urolithiasis. Both patients had positive preoperative urine cultures for Candida albicans and had been treated with prolonged courses of broad-spectrum antibiotics before ureteroscopy [27]. Nicholson et al. reported systemic candidiasis after removal of a ureteral stent in a renal transplant recipient. The stent was removed despite growth of Candia albicans in urine culture without antifungal treatment, but the stent was not investigated [28]. Another example is the report of Riedel et al. about severe infectious complications after stent manipulation in patients with positive urine cultures prior to the urological procedure [5]. The severe clinical consequences of these reports highlight (1) the need for preinterventional UTI screening in immunocompromised patients and (2) the identification of Candida albicans as major causative pathogen in severe infectious complications associated with ureteral stent manipulation. The limitation of urine culture in predicting MUSC has been reported previously [10, 16-18] and was confirmed by our study. However, this is not surprising because urine culture can only detect free-floating (planktonic) organisms. As MUSC is infrequently associated with both positive urine culture and infectious complications, the routine application of antibiotic prophylaxis in asymptomatic, healthy patients with indwelling stents prior to cystoscopy or device removal is debatable.

The most important clinical conclusion of our study is that the roll-plate technique appears to be superior to sonication in the detection of MUSC. Whenever a clinical 
Fig. 2 Microbiological results of roll-plate versus sonication. a Number of microorganisms isolated by sonication and rollplate. b Percentage of microorganisms isolated matched to sonication and rollplate $a$ (sonication), $b$ (rollplate) $1 \mathrm{~A}-$ Aerococcus urinae $(n=2)$, Actinobaculum Schalii $(n=1)$, Streptococcus spp. $(n=2)$, Gram-labile rods $(n=2)$, Gram-positive rods $(n=1) ; 1 \mathrm{~B}$-Aerococcus urinae $(n=2)$, Actinobaculum Schalii $(n=1)$, Streptococcus spp. $(n=2)$, Gram-labile rods $(n=2)$, Gram-positive rods $(n=1) .2 \mathrm{~A}-$ Escherichia coli $(n=9)$, Klebsiella spp. $(n=3)$, Proteus spp. $(n=3)$, Serratia marcescens $(n=1)$; 2B-Escherichia coli $(n=12)$, Klebsiella spp. $(n=4)$, Proteus spp. $(n=4)$, Serratia marcescens $(n=2)$ situation requires the diagnosis of MUSC, the clinician can rely on the results obtained by the simple and cost-effective roll-plate technique. Sonication, which often is not routinely available in the microbiological laboratory, remains a valuable tool for the diagnosis of prosthetic joint infections, but is not necessary for the diagnosis of MUSC [29].

Despite the advantages of roll-plate compared with sonication, the ideal method for the investigation of MUSC has yet to be found. In this context, microcalorimetry appears promising, as this method allows the investigation of the whole bacterial population on the stent instead of mechanically detachable organisms only [30].

\section{Conclusion}

This is the first prospective randomised study to demonstrate that Maki's roll-plate technique is superior to sonication in the detection of MUSC, and urine culture is less
(A) Number of microorganisms isolated by sonication and roll-plate

(B) Percent of microorganisms isolated matched to sonication and roll-plate

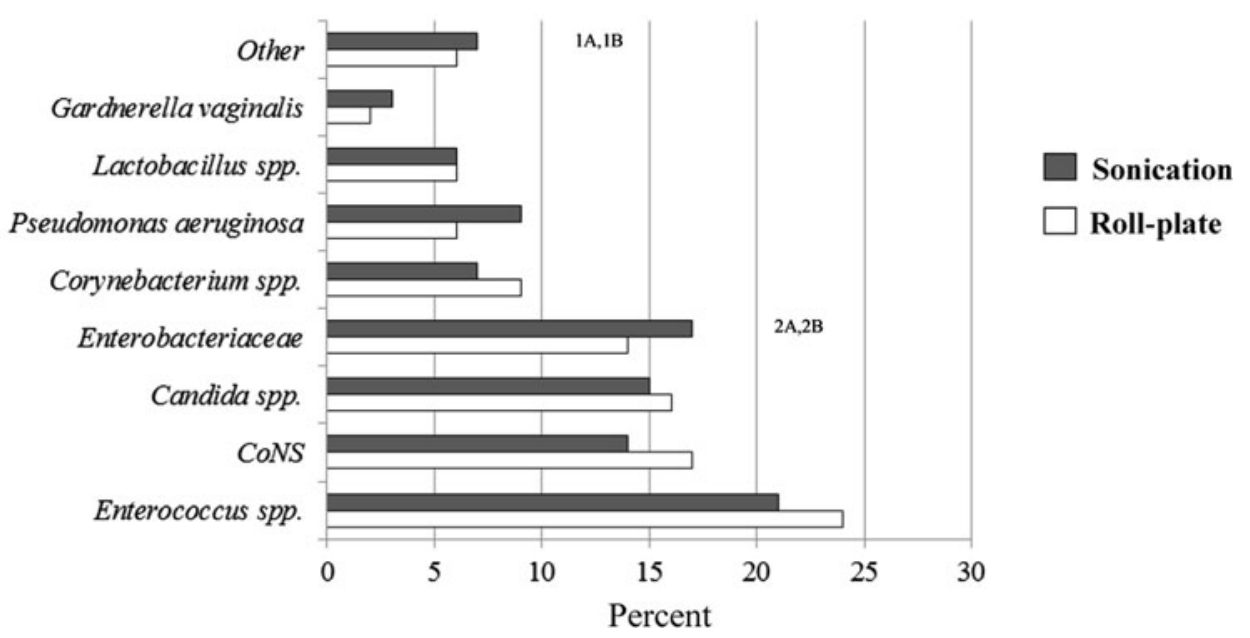

sensitive than both methods. Maki's technique should be regarded as the diagnostic procedure of choice for studying MUSC for both routine clinical investigation and basic laboratory research.

Conflict of interest None of the contributing authors has any conflict of interest relevant to the subject matter or materials discussed in the manuscript. No funding or other financial support was received.

\section{References}

1. Joshi HB, Stainthorpe A, MacDonagh RP, Keeley FX Jr, Timoney AG, Barry MJ (2003) Indwelling ureteral stents: evaluation of symptoms, quality of life and utility. J Urol 169: 1065-1069

2. Richter S, Ringel A, Shalev M, Nissenkorn I (2000) The indwelling ureteric stent: a 'friendly' procedure with unfriendly high morbidity. BJU Int 85:408-411

3. Bonkat G, Rieken M, Muller G, Roosen A, Siegel FP, Frei R, Wyler S, Gasser T, Bachmann A, Widmer AF (2012) Microbial 
colonization and ureteral stent-associated storage lower urinary tract symptoms: the forgotten piece of the puzzle? World J Urol

4. Gautam G, Singh AK, Kumar R, Hemal AK, Kothari A (2006) Beware! Fungal urosepsis may follow endoscopic intervention for prolonged indwelling ureteral stent. J Endourol 20:522-524

5. Riedl CR, Plas E, Hubner WA, Zimmerl H, Ulrich W, Pfluger $H$ (1999) Bacterial colonization of ureteral stents. Euro Urol 36:53-59

6. Dellis A, Joshi HB, Timoney AG, Keeley FX Jr (2010) Relief of stent related symptoms: review of engineering and pharmacological solutions. J Urol 184:1267-1272

7. Lange D, Elwood CN, Choi K, Hendlin K, Monga M, Chew BH (2009) Uropathogen interaction with the surface of urological stents using different surface properties. J Urol 182:1194-1200

8. Mendez-Probst CE, Fernandez A, Denstedt JD (2010) Current status of ureteral stent technologies: comfort and antimicrobial resistance. Curr Urol Rep 11:67-73

9. Mendez-Probst CE, Goneau LW, Macdonald KW, Nott L, Seney S, Elwood CN, Lange D, Chew BH, Denstedt JD, Cadieux PA (2012) The use of triclosan eluting stents effectively reduces ureteral stent symptoms: a prospective randomized trial. BJU Int 110:749-754

10. Bonkat G, Rieken M, Rentsch CA, Wyler S, Feike A, Schafer J, Gasser T, Trampuz A, Bachmann A, Widmer AF (2011) Improved detection of microbial ureteral stent colonisation by sonication. World J Urol 29:133-138

11. Bonkat G, Rieken M, Siegel FP, Frei R, Steiger J, Groschl I, Gasser TC, Dell-Kuster S, Rosenthal R, Gurke L, Wyler S, Bachmann A, Widmer AF (2012) Microbial ureteral stent colonization in renal transplant recipients: frequency and influence on the short-time functional outcome. Transpl Infect Dis 14:57-63

12. Paick SH, Park HK, Oh SJ, Kim HH (2003) Characteristics of bacterial colonization and urinary tract infection after indwelling of double-J ureteral stent. Urology 62:214-217

13. Reid G, Denstedt JD, Kang YS, Lam D, Nause C (1992) Microbial adhesion and biofilm formation on ureteral stents in vitro and in vivo. J Urol 148:1592-1594

14. Tieszer C, Reid G, Denstedt J (1998) Conditioning film deposition on ureteral stents after implantation. J Urol 160:876-881

15. Klis R, Korczak-Kozakiewicz E, Denys A, Sosnowski M, Rozanski W (2009) Relationship between urinary tract infection and self-retaining Double-J catheter colonization. J Endourol 23: 1015-1019

16. Farsi HM, Mosli HA, Al-Zemaity MF, Bahnassy AA, Alvarez M (1995) Bacteriuria and colonization of double-pigtail ureteral stents: long-term experience with 237 patients. J Endourol 9:469-472

17. Lifshitz DA, Winkler HZ, Gross M, Sulkes J, Baniel J, Livne PM (1999) Predictive value of urinary cultures in assessment of microbial colonization of ureteral stents. J Endourol 13:735-738
18. Lojanapiwat B (2006) Colonization of internal ureteral stent and bacteriuria. World J Urol 24:681-683

19. Maki DG, Weise CE, Sarafin HW (1977) A semiquantitative culture method for identifying intravenous-catheter-related infection. N Engl J Med 296:1305-1309

20. Kehinde EO, Rotimi VO, Al-Hunayan A, bdul-Halim H, Boland F, Al-Awadi KA (2004) Bacteriology of urinary tract infection associated with indwelling $\mathrm{J}$ ureteral stents. $\mathrm{J}$ Endourol 18:891-896

21. Bouza E, Alvarado N, Alcala L, Sanchez-Conde M, Perez MJ, Munoz P, Martin-Rabadan P, Rodriguez-Creixems M (2005) A prospective, randomized, and comparative study of 3 different methods for the diagnosis of intravascular catheter colonization. Clin Infect Dis 40:1096-1100

22. Slobbe L, El BA, Boersma E, Rijnders BJ (2009) Comparison of the roll plate method to the sonication method to diagnose catheter colonization and bacteremia in patients with long-term tunnelled catheters: a randomized prospective study. J Clin Microbiol 47:885-888

23. Mermel LA, Allon M, Bouza E, Craven DE, Flynn P, O’Grady NP, Raad II, Rijnders BJ, Sherertz RJ, Warren DK (2009) Clinical practice guidelines for the diagnosis and management of intravascular catheter-related infection: 2009 Update by the Infectious Diseases Society of America. Clin Infect Dis 49:1-45

24. Wilson ML, Gaido L (2004) Laboratory diagnosis of urinary tract infections in adult patients. Clin Infect Dis 38:1150-1158

25. Keane PF, Bonner MC, Johnston SR, Zafar A, Gorman SP (1994) Characterization of biofilm and encrustation on ureteric stents in vivo. Br J Urol 73:687-691

26. Hola V, Ruzicka F, Horka M (2010) Microbial diversity in biofilm infections of the urinary tract with the use of sonication techniques. FEMS Immunol Med Microbiol 59:525-528

27. Gross M, Winkler H, Pitlik S, Weinberger M (1998) Unexpected candidemia complicating ureteroscopy and urinary stenting. Eur J Clin Microbiol Infect Dis 17:583-586

28. Nicholson ML, Veitch PS, Donnelly PK, Bell PR (1992) Systemic candidiasis caused by a renal transplant ureteric stent. Br J Urol 70:448-449

29. Trampuz A, Piper KE, Jacobson MJ, Hanssen AD, Unni KK, Osmon DR, Mandrekar JN, Cockerill FR, Steckelberg JM, Greenleaf JF, Patel R (2007) Sonication of removed hip and knee prostheses for diagnosis of infection. N Engl J Med 357:654-663

30. Bonkat G, Braissant O, Widmer AF, Frei R, Rieken M, Wyler S, Gasser TC, Wirz D, Daniels AU, Bachmann A (2012) Rapid detection of urinary tract pathogens using microcalorimetry: principle, technique and first results. BJU Int 110:892-897 